\title{
Evidence of transverse magnetospheric field line oscillations as observed from Cluster and ground magnetometers
}

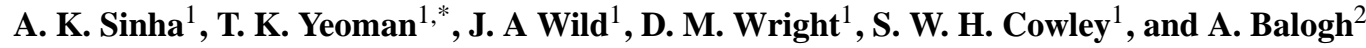 \\ ${ }^{1}$ Department of Physics and Astronomy, University of Leicester, LE1 7RH. UK \\ ${ }^{2}$ Imperial College, London, UK \\ *Permanent address: Indian Institute of Geomagnetism, Mumbai, India
}

Received: 2 February 2004 - Revised: 31 January 2005 - Accepted: 2 February 2005 - Published: 30 March 2005

\begin{abstract}
The dynamic spectrum of ULF waves from magnetic field data obtained by the elliptically orbiting Cluster satellites (with an apogee of $119000 \mathrm{~km}$, perigee of $19000 \mathrm{~km}$ and the orbital period of $57 \mathrm{~h}$ ) have been prepared in the frequency range 0 to $120 \mathrm{mHz}$ when the satellite was near its perigee. The existence of field line oscillations, with increasing frequency in the inbound sector and decreasing frequency in the outbound sector, is seen in the transverse components, indicating the presence of independently oscillating local magnetic flux tubes in the form of transverse standing Alfvén waves. The results show that toroidal and poloidal modes are excited simultaneously. The analysis of simultaneous ground magnetometer data at the footprint of the satellite suggests that these modes are also excited due to coupling to magnetospheric waveguide modes. The clear signature of a resonant fundamental mode is seen in the ground data whereas Cluster detects a harmonic of this frequency. Lower frequency modes indicative of waveguide oscillations are seen in both the ground data and the compressional field at Cluster.
\end{abstract}

Keywords. Magnetospheric physics (Magnetosphereionosphere interactions; Magnetospheric configuration and dynamics; MHD waves and instabilities)

\section{Introduction}

Observations of long period geomagnetic pulsations (10 s$600 \mathrm{~s}$ period) in the Earth's magnetic field have a long history of almost a century. The concept of an independent field line oscillation was introduced by Dungey (1954) who proposed for the first time that the long-period geomagnetic pulsations observed on the ground might be the result of standing Alfvén waves being excited on geomagnetic field lines. The concept has there after taken deep roots and there are many ground based and satellite observations that have estab-

Correspondence to: T. K. Yeoman

(tim.yeoman@ion.le.ac.uk) lished the relevance of field line oscillations (e.g. Cummings et al., 1969; Anderson et al., 1990). A number of theoretical estimates of field line eigenperiods are available at middle and high latitudes where hydrogen is dominant plasma component (e.g. Cummings et al., 1969; Sinha and Rajaram, 1997) as well as at low and equatorial latitudes where heavier species such as oxygen and helium take over (e.g. Poulter et al., 1988; Sinha et al., 2002).

In satellite data (e.g. Anderson et al., 1990), a dynamic spectrum of geomagnetic field oscillations showed a clear increase of the frequency in the in-bound sector of the orbit and a decrease in the frequency in the out-bound sector consistent with the expectations from the field line resonance model. Such field line resonance signatures have been detected by the AMPTE/CCE satellite, right from 8 Earth radii $\left(R_{E}\right)$, to almost $2 R_{E}$ (Takahashi and Anderson, 1992). Field line resonance in ground geomagnetic data is determined by the frequency spectra at the foot of the field line. The signal corresponding to the characteristic resonance period of the field line will maximize on ground at the latitude corresponding to the footprint of the field line (Menk et al., 1994).

Global compressional oscillations of the magnetosphere, setting the cavity formed by magnetospheric boundaries into oscillation, might cause the local field line to resonate at its characteristic frequency as suggested by Kivelson et al. (1984). Using a box model for the magnetosphere Kivelson and Southwood $(1985,1986)$ and Zhu and Kivelson (1988) tried to explain discrete frequencies of pulsations as seen in the ground-based observations and concluded that resonant field line oscillations might be explained in terms of coupling between the local field lines and the global cavity modes. Detailed analytical and numerical studies using hemicylindrical magnetospheric model were presented in a series of papers by Allan et al. (1985, 1986a,b, 1987a,b). The slower decay of resonant modes than those expected due the ionospheric Joule dissipation as reported by Crowley et al. $(1987,1989)$ was attributed to a continued supply of energy from a global cavity oscillation. More recently Harrold and Samson (1992), Samson et al. (1992), Walker 
et al. (1992), Wright (1994), Rickard and Wright (1994) and Walker (2000) have suggested that the magnetospheric cavity oscillation is better described as a magnetospheric waveguide, with the characteristic frequencies of the waveguide coupling to field line oscillations.

As far as variation of pulsation frequency with latitude on ground is concerned, several workers (Samson et al., 1971; Samson and Rostoker, 1972; Walker et al., 1979) have observed many events for which the pulsation frequency is same at all latitudes with the latitude corresponding to maximum amplitude varying with the period. These events correspond to resonances which occurred at a single frequency throughout and the region of maximum amplitude represented the resonance of field lines with a nearly monochromatic driving source. These observations were consistent with the theoretical predictions of Chen and Hasegawa (1974a) and Southwood (1974). The class of pulsations in which the frequency varies with latitude (Poulter and Neilson, 1982; Engebretson et al., 1986) are also consistent with these theoretical concepts, except that field lines are simultaneously oscillating at their natural frequencies over a range of latitudes. The former type of pulsations with constant frequency could be associated with monochromatic driver while those in the latter case with latitude dependent frequencies could be attributed to an impulsive source (Allan and Poulter, 1984). Observations from AMPTE/CCE showed that the pulsations exhibiting continuous variation of frequency with $L$-shell were long-lived and the results did not go well with the concept of an impulsive driving source (Anderson et al., 1989). Events displaying discrete resonant frequencies depicting neither monochromatic nor continuous driving source were reported by Rostoker and Samson (1972), while McDiarmid and Nielsen (1987) reported the simultaneous occurrence of monochromatic and variable frequency events. Continuous simultaneous excitation of harmonics at a particular $L$-shell were also observed and were associated with a broadband driving source (Takahashi and McPherron, 1984). Thus the monochromatic and impulsive source hypotheses alone could not explain the features of observed pulsations.

Taking into account the effects of spatial integration, Poulter and Allan (1985) demonstrated that the pulsations observed by satellite showing latitudinal variations in frequency and those observed in the ground showing constant frequency with the latitude could belong to the same class. Using empirical models of ionospheric currents they modelled ground magnetic signatures of transient pulsation events and showed that on the ground we normally observe a reduced variation of frequency with latitude. By extensive modelling of the data considering the effects of spatial integration using empirical models of ionospheric currents they found that on the ground at the latitudes not located at the site of greatest pulsation amplitudes, the period of oscillations appears nearly constant even though the actual pulsation period varies continuously with latitude. They demonstrated that in all cases the period observed by ground magnetometers are skewed towards the oscillation period of greatest intensity.
In this investigation we present the first results from the Cluster satellites which clearly demonstrate that the standing Alfvén waves excited on freely oscillating geomagnetic field lines could be a viable mechanism for the observed pulsations in the ULF range. The increase in the wave frequency in the inbound sector and decrease in that in the outbound sector as seen in the dynamic spectrum of geomagnetic field variation is typical of the characteristics of standing Alfvén waves (Anderson et al., 1990). An analysis of the simultaneous ground magnetometer data when the footprint of the satellite were passing through the IMAGE-network sector has also been performed. Although we see a latitudinal variation of the pulsation frequency in the satellite observations, the ground data show a constant, and longer period with respect to latitude. Evidence of these longer periods is also seen on Cluster, and the simultaneous observations of the satellite and ground magnetometers suggest that these oscillations could represent field line oscillations excited through coupling to harmonics of waveguide oscillations.

In Sect. 2 we present the results from the Cluster satellite observations, and in Sect. 3 the results from simultaneous ground data at the approximate footprint of the satellite are discussed. Finally, in Sect. 4 we summarize and conclude our findings in the perspective of field line oscillations as a plausible explanation for the observed geomagnetic pulsations.

\section{Satellite observations}

The Cluster mission comprises of four identical spacecraft launched into elliptical polar orbits around the earth with an apogee of $119000 \mathrm{~km}$, perigee of $19000 \mathrm{~km}$ and the orbital period of $57 \mathrm{~h}$. Fluxgate Magnetometers located on a $5 \mathrm{~m}$ long boom to avoid any interference from the spacecraft, measure the magnetic field along the orbit and they can take high resolution measurements with up to 67 samples per second.

For our present studies we deal with the magnetic field data averaged over $4 \mathrm{~s}$. The data were archived in the Geocentric Solar Ecliptic (GSE) coordinate system. Using the appropriate transformation (Hapgood, 1992) the variables were transformed to the Geomagnetic (MAG) coordinate system where $Z$-axis coincides with the dipole axis, the $Y$ axis is the line of intersection between geographic equator and the geographic meridian $90^{\circ} \mathrm{E}$ of the meridian containing the dipole axis and the $X$-axis is defined by right-handed orthogonal system $(X, Y, Z)$. The variables were then transformed to a local dipole coordinate system (LDC), where LDC $Z$ is the direction along the local dipole field, LDC $Y$ is eastward, perpendicular to the plane containing LDC $Z$ and the diople, and LDC $X$ makes up the right-handed set, pointing outwards. Thus, for a dipole-like field LDC $X$ will contain transverse poloidal oscillations of the magnetic field, LDC $Y$ transverse toroidal oscillations, and LDC $Z$ is the compressional component.

After subtracting the Tsyganenko-96 model from the magnetic field data they were subjected to a filter above the 

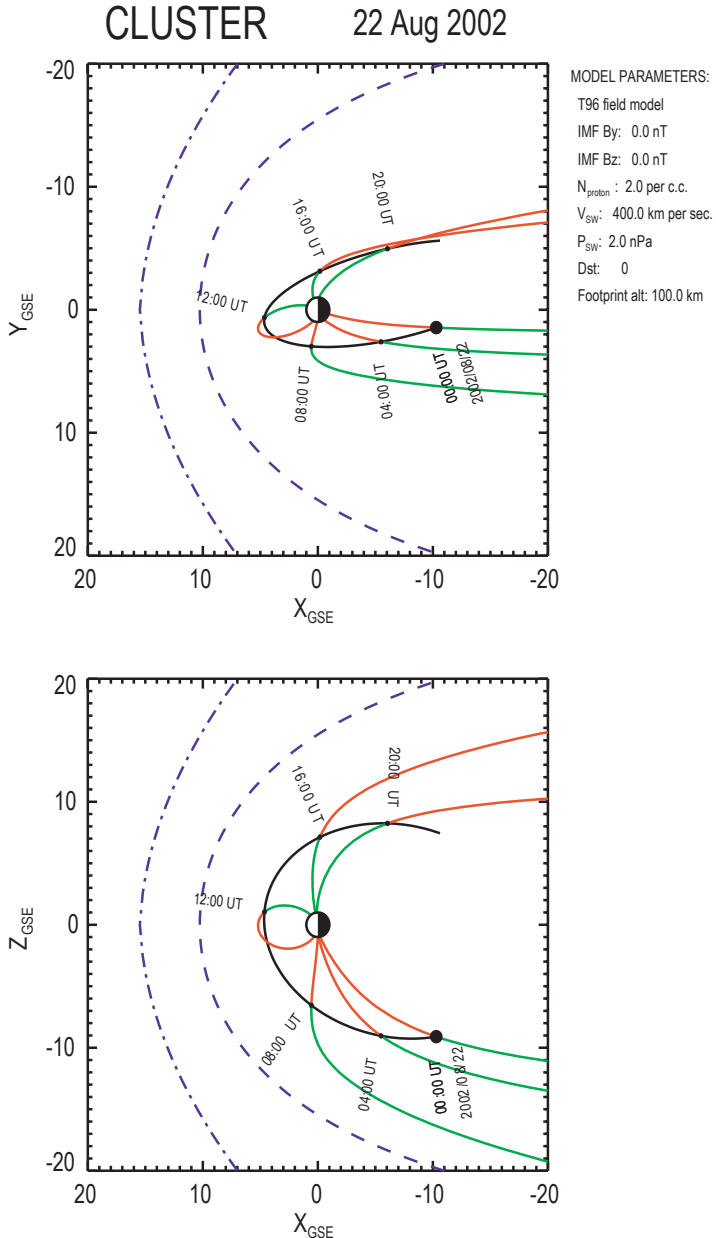

Fig. 1. The orbit of the cluster satellites on 22 August 2002 in GSE coordinates, indicating that during 10:00-12:00 UT the satellites cross the dipolar field lines in the inner magnetosphere.

periods of $5 \mathrm{~s}$. Dynamic spectra were constructed by taking intervals of 256 data points spaced at $4.0 \mathrm{~s}$ intervals for a data interval of around $17 \mathrm{~min}$, reducing to zero mean, applying a $10 \%$ cosine bell taper at the edges of the window, and applying a Fourier transform. Each subsequent transform was shifted by 30 data points $(2 \mathrm{~min})$. The power thus obtained were normalized with respect to the maximum power in the spectrum. In the dynamic spectra presented the color bar to the right of the spectrum shows the log of the normalized power.

It should be noted that unlike AMPTE/CCE which was orbiting close to the equator, Cluster is a polar satellite. In the data we have chosen, the satellite tracks the magnetic field corresponding to a very high $L$-value to $\sim L=4$ and back to very high value of $L$ within a time of around $2 \mathrm{~h}$. An examination has been made of the magnetic field data of four months of the year 2002 when the satellite was in the inner magnetosphere near its perigee. For the present paper we concentrate on a particular event of 22 August 2002 when the spacecraft was crossing the dipolar region of the inner mag-

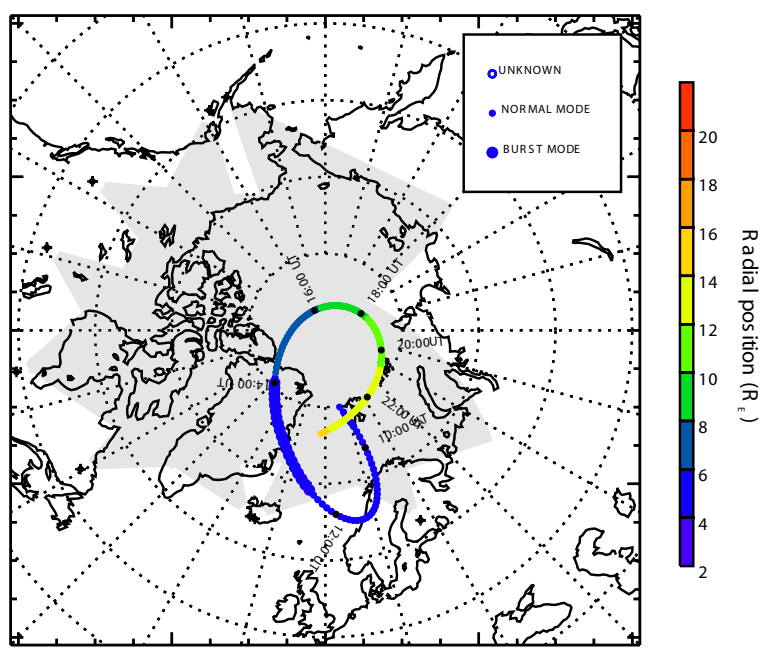

Fig. 2. The footprint of the cluster satellites on 22 August 2002. The radial geocentric position of the spacecraft is colour-coded according to the scale on the right. The footprint is calculated using the Tsyganenko 96 magnetic field model (solar wind pressure $2 \mathrm{nPa}$, $B_{y}=B_{z}=0 \mathrm{nT}, D_{s t}=-50 \mathrm{nT}$ ). The cluster footprint overlies the IMAGE magnetometer chain during 10:00-12:00 UT.

netosphere. The orbit is presented in Fig. 1. Figure 2 shows the footprint location of the satellite corresponding to the orbit shown in Fig. 1, with the radial geocentric position of the spacecraft colour-coded according to the scale on the right. The footprint is calculated using the Tsyganenko $96 \mathrm{mag}$ netic field model (solar wind pressure $2 \mathrm{nPa}, B_{y}=B_{z}=0 \mathrm{nT}$, $D_{s t}=-50 \mathrm{nT}$ ). Our focus is on the region when the footprint location falls in the region of the IMAGE network of ground magnetometers, so that we can look at the spacecraft data in the context provided by ground-based instrumentation. Figure 3 shows a projection of a field line tracing into the GSE $X Z$ plane, using the same magnetic field model as presented in Fig. 2, from the typical satellite location with respect to ground stations of the IMAGE network at 11:15 UT. The detailed coordinates of these stations are shown in Table 1, with the field line traces for the IMAGE stations in Fig. 3 shown in a variety of dashed and dot-dashed line styles. The solid circles coloured in blue, green, yellow and red represent satellites 1,2, 3 and 4, respectively. The field line has been traced as a solid line for satellite 1 and the relative positions of the other satellites at 11:15 UT have been plotted with respect to the IMAGE magnetometer station field lines. For this event we are perfectly placed to perform an analysis from the combined perspective of in-situ measurements by the onboard satellite magnetometers and simultaneous ground measurements by IMAGE magnetometer network.

The spectrogram for this particular event is presented in Fig. 4. The first three panels in this figure present the local dipole $X, Y$ and $Z$ components, whereas the bottom panel presents the satellite $L$-shell. Though the lower harmonics are suppressed by the short timeseries lengths in the spectrograms, and will be discussed later, the continuous rising and 
Table 1. Coordinates of stations.

\begin{tabular}{|c|c|c|c|c|c|}
\hline Abv. & Name & $\begin{array}{c}\text { G. } \\
\text { lat. }^{\circ} \mathrm{N}\end{array}$ & $\begin{array}{c}\mathrm{G} . \\
\text { long. }{ }^{\circ} \mathrm{E}\end{array}$ & $\begin{array}{l}\text { CGM. } \\
\text { lat. }^{\circ} \mathrm{N}\end{array}$ & $\begin{array}{l}\text { CGM. } \\
\text { long. }{ }^{\circ} \mathrm{E}\end{array}$ \\
\hline NAL & Ny Ålesund & 78.9 & 11.95 & 75.25 & 112.08 \\
\hline HOR & Hornsund & 77.00 & 15.60 & 74.13 & 109.59 \\
\hline BJN & Bear Island & 74.50 & 19.20 & 71.45 & 108.07 \\
\hline SOR & Sørøya & 70.54 & 22.22 & 67.34 & 106.17 \\
\hline TRO & Troms $\varnothing$ & 69.66 & 18.94 & 66.64 & 102.90 \\
\hline KIL & Kilpisjärvi & 69.02 & 20.79 & & 103.79 \\
\hline MUO & Muonio & 68.02 & 23.53 & 64.72 & 105.22 \\
\hline PEL & Pello & 66.90 & 24.08 & 63.55 & 104.92 \\
\hline OUJ & Oulujärvi & 64.52 & 27.23 & 60.99 & 106.14 \\
\hline HAN & Hankasalmi & 62.30 & 26.65 & 58.71 & 104.61 \\
\hline
\end{tabular}

SATELLITE POSITION ON 22 AUG 2002 AT 11:15 UT

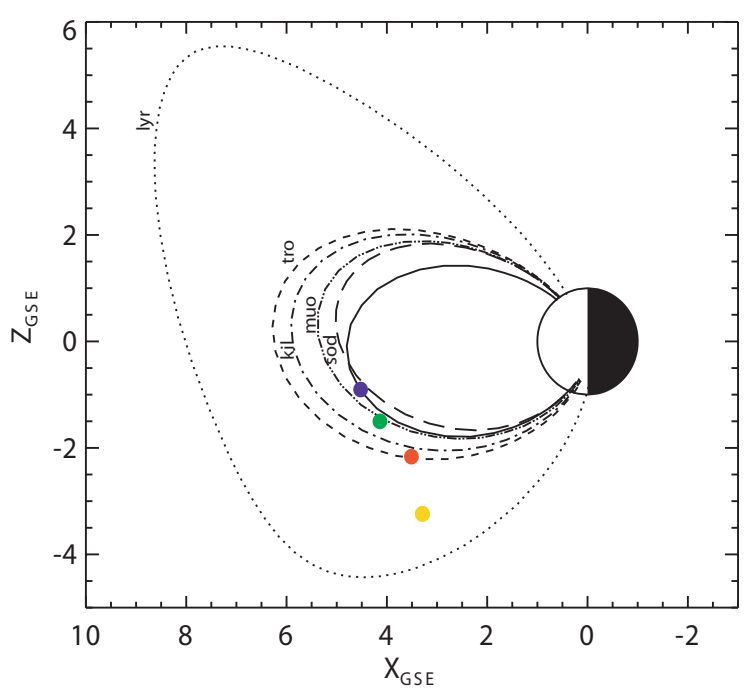

Fig. 3. Satellite positions with respect to geomagnetic field lines projected into the GSE $X Z$ plane corresponding to IMAGEnetwork stations on 22 August 2002 at 11:15 UT. The field is calculated using the Tsyganenko 96 magnetic field model (solar wind pressure $2 \mathrm{nPa}, B_{y}=B_{z}=0 \mathrm{nT}, D_{s t}=-50 \mathrm{nT}$ ). Solid circles in blue, green, yellow and red represent the positions of spacecraft $1,2,3$ and 4 , respectively. The field line has been traced for spacecraft 1 (solid line), the IMAGE stations (dashed and dot-dashed lines), and the relative positions of the other Cluster spacecraft have been shown.

falling of bright tones in the inbound and outbound sectors of the satellite are quite evident for higher harmonics in the transverse components (LDC $X$ and $Y$ ). These signatures are typical and unique to standing Alfvén waves for which the frequency should increase with a decrease in $L$-value and vice-versa (Anderson et al., 1990). Both toroidal (LDC Y) oscillations and poloidal (LDC $X$ ) mode oscillations are observed. It should be noted that we see such signatures consistently on a number of days when the satellite was crossing the dipolar region of inner magnetosphere, although here we are present a typical case study when the footprint of the satel- lite overlies in the IMAGE magnetometer network so that by analyzing simultaneous ground data we can examine if these oscillations cast their signatures in the ground observations.

Apart from freely ringing field line oscillations showing latitude dependent features in all the components, a discrete "hot-spot" of higher power at $\sim 27 \mathrm{mHz}$ frequency is observed in the transverse components of Fig. 4 at 11:15 UT. This is clearly a different phenomenon than just the natural freely ringing oscillations and points to the coupling of field line oscillations to a narrow-band energy source at that time. A more detailed examination of the frequency content of the Cluster data during the "hot spot" interval is given in Fig. 5, which presents a static spectrum of the three field components and the total field between 10:45 and 11:30 UT. Each static spectrum presented in this paper is produced by subtracting the Tsyganenko-96 field (for the Cluster data), reducing the timeseries to zero mean, bandpass-filtering, (here between 300 and $5 \mathrm{~s}$ ), then applying a $10 \%$ cosine bell taper. The $27 \mathrm{mHz}$ hot spot itself is a clear feature of the transverse components of this static spectrum, and some additional higher harmonics are also visible. Lower frequency power is observed at $\sim 6 \mathrm{mHz}$ in all components, with additional peaks in the $10-16 \mathrm{mHz}$ range. The longer spectrum interval used thus reveals long period, lower harmonic activity than is seen in the dynamic spectrum, and wave activity in the compressional as well as transverse components. The static spectra shown in Fig. 5 clearly demonstrate the existence of structured wave signatures at lower frequencies than those seen most clearly in the dynamic spectral analysis of Fig. 4. This is particularly clear in the 10:00-11:00 UT region of Fig. 4, where considerable low-frequency power is observed in the transverse components. To investigate this region in more detail a timeseries of the three LDC components and the total field from 10:00 and 12:00 UT, bandpass filtered between 1000 and $5 \mathrm{~s}$, is presented in Fig. 6. In this figure the high frequency $27 \mathrm{mHz}$ "hot spot" is seen in the LDC $X$ and $Y$ components at 11:06 UT, and the presence of $6 \mathrm{mHz}$ waves can be seen just before this. Clear evidence of lower frequency wave activity is seen in the compressional components. Spectral analysis of this data, shown in Fig. 7 reveals a harmonic series of compressional waves at frequencies of $0.8,1.4,1.9,2.9$, and $3.8 \mathrm{mHz}$. A further clear peak is seen at close to $6 \mathrm{mHz}$, but this peak is clearest in the azimuthal transverse component. Again some strong higher harmonics are also observed. The cluster spacecraft thus observes a complex range of wave signatures, ranging from low-frequency apparently latitude-independent waves, through discrete latitude-independent waves of intermediate frequency observed in both compressional and transverse components, through to higher-frequency latitude dependent spectral features confined to the transverse components. An interpretation of the spectral features observed, however, requires an examination of ground magnetograms around the conjugate point of the Cluster spacecraft. 


\section{Cluster Dynamic Power Spectra, S pacecraft 2}

\section{CLUSTER 2 (Salsa), 22- 8- 2002}
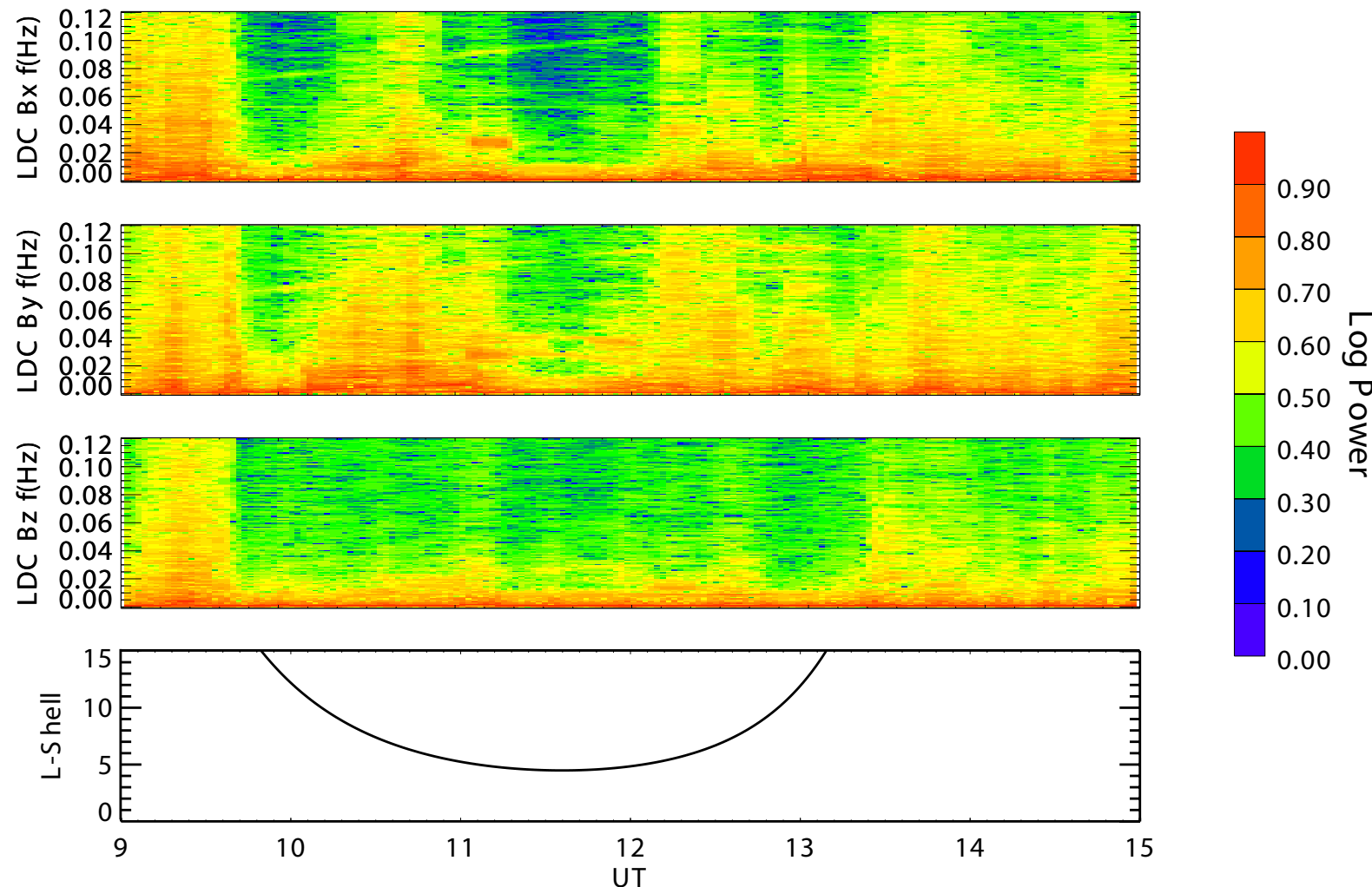

Fig. 4. The dynamic power spectrum of magnetic field data from spacecraft 2 of cluster for 09:00-15:00 UT. The upper, three panels represent magnetic local dipole coordinate (LDC) $X, Y$ and $Z$ components respectively (the coordinate system is been described in the text), with normalised logarithmic Fourier spectral power being represented by a colour scale. The bottom panel shows the spacecraft $L$-value.

\section{Ground data}

The field line oscillations seen at Cluster should also manifest themselves through magnetic field variations at least at ionospheric heights. IMAGE (International Monitor for Auroral Geomagnetic Effects) is a network of chain of 27 magnetometer stations covering geographic latitudes from $58^{\circ}$ to $79^{\circ}$. Data sampled at $10 \mathrm{~s}$ intervals at the magnetometer stations shown in Table 1 were subjected to spectral analysis when the footprints of the satellite were in the region of network stations. The data were available in the conventional $X$, $Y$ and $Z$ geographic coordinate system, which for the Scandinavian meridian is virtually identical to magnetic coordinates.

\subsection{Field line oscillations}

The $X$ and $Y$ variations recorded at the IMAGE stations in Table 1 between 11:30 and 12:00 UT on 22 August 2002 were bandpass filtered between $300 \mathrm{~s}$ and $20 \mathrm{~s}$ period, and are shown in Fig. 8. A clear wavepacket can be observed with a maximum amplitude in the PEL and MUO $X$ components. The filtered data were then subjected to Fourier analysis, revealing a clear spectral peak in the $X$ compo- nent at frequencies of $6.1-6.6 \mathrm{mHz}$, with additional higher frequency peaks seen at the lowest latitudes, and lower frequency peaks seen at the highest latitudes. A latitudinal profile of the Fourier power and phase for the spectral maxima at $6.1 \mathrm{mHz}$ is presented in Fig. 9, revealing a broad latitudinal amplitude peak centred at PEL at this frequency, and a corresponding slow latitudinal phase variation in Fourier phase of $\sim 150^{\circ}$. Thus, there is evidence for field line resonance activity on the ground at a frequency corresponding to the $6 \mathrm{mHz}$ spectral peak observed at Cluster.

\subsection{Waveguide oscillations}

The cavity/waveguide formed by magnetospheric boundaries could be set into oscillations by global compression or magnetosheath flow (Mann et al., 1999) and that could cause the local field line to oscillate at its characteristic frequency. This idea is supported by extensive theoretical analysis, as detailed in the introduction. It will be interesting to see if such modes were in operation during the event. That will throw some light if field line oscillations could be excited via the coupling to waveguide modes.

Having applied a band-pass filter between the periods of $1000 \mathrm{~s}$ and $20 \mathrm{~s}$, the $X$ and $Y$ components of ground magnetic 


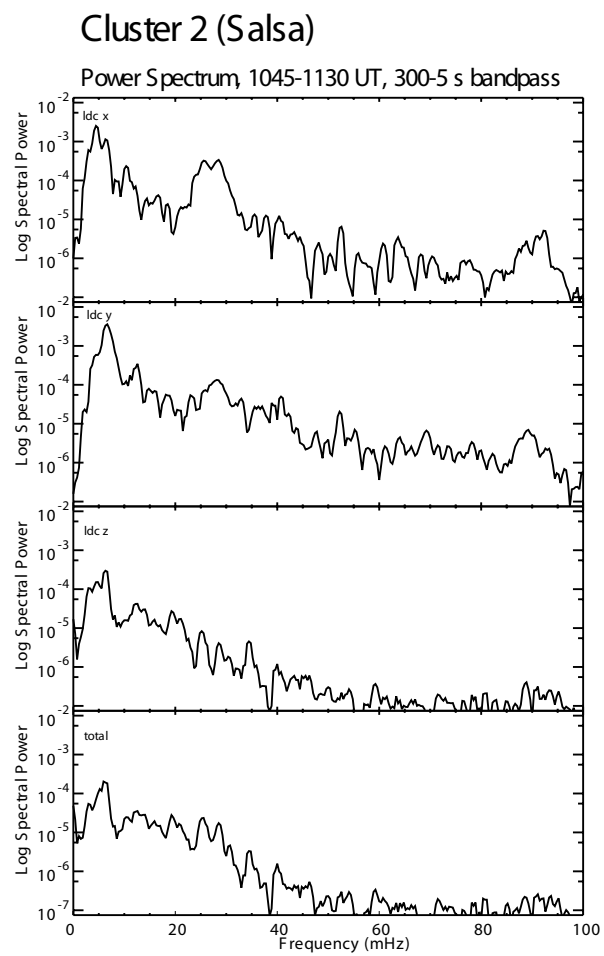

Fig. 5. A Fourier spectrum of the LDC $X, Y, Z$ component and total magnetic field from Cluster 2, from 10:45-11:30 UT, bandpass filtered between $300-5 \mathrm{~s}$. Power for each component is indicated on a common logarithmic scale.

data between 10:00 to 12:00 UT were subjected to spectral analysis. The filtered data for the $X$ and $Y$ components are shown in Fig. 10. The most obvious feature apparent in these time series is the strong $X$ component wave signatures at the highest latitudes of the IMAGE array, although the higher frequency wave activity observed at lower latitudes around 11:45 UT, presented in more detail in Fig. 8, is also visible. Fourier analysis of the $X$ component magnetograms is presented in Fig. 11. A clear set of harmonic peaks is observed over the magnetometer array at frequencies of $0.8,1.4,1.9$, 2.9 , and $3.7 \mathrm{mHz}$, exactly the same harmonic series as observed on Cluster, discussed above and shown in Fig. 7. The $1.9 \mathrm{mHz}$ peak is the dominant one over most of the array, although the $1.4 \mathrm{mHz}$ peak is the largest at the two highest latitude stations. The $0.8 \mathrm{mHz}$ peak is small, and most obvious at the highest and lowest latitudes of the array. Higher frequency peaks are also visible at low power in the spectra, including the $6 \mathrm{mHz}$ peak centred at MUO, which was discussed above. A latitudinal profile of the Fourier power and phase for the spectral maxima at $1.9 \mathrm{mHz}$, which dominates over most of the array, is presented in Fig. 12. A characteristic amplitude peak and $180^{\circ}$ phase change can be observed in the $X$ component magnetograms, at the highest latitudes of the IMAGE array. Thus there is evidence of driven field line resonance behaviour at high latitude for the $1.9 \mathrm{mHz}$ peak.
Cluster 2 (Salsa)

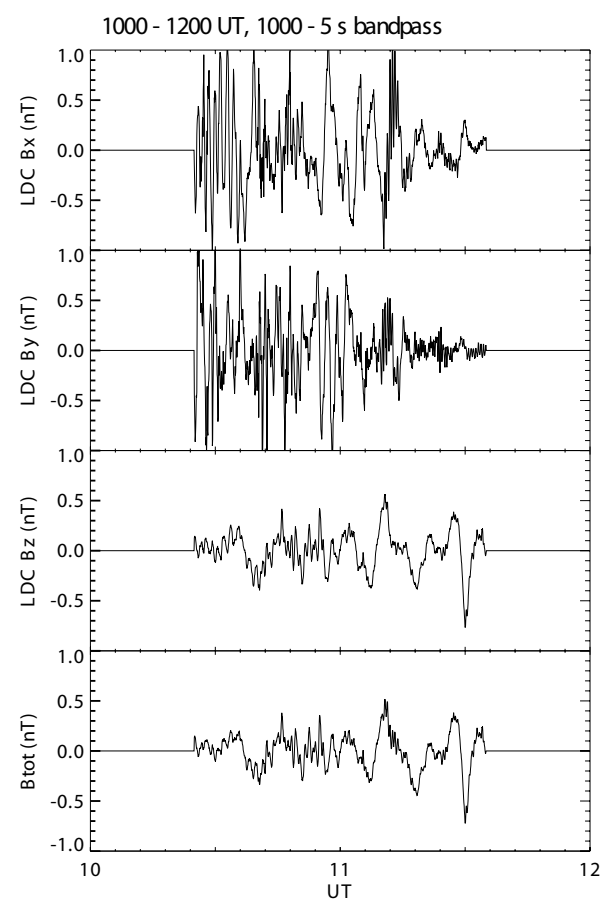

Fig. 6. Components of magnetic field data in LDC $X, Y, Z$ component and total magnetic field as observed from spacecraft 2 of cluster for 10:00-12:00 UT after subtracting Tsyganenko-96 and bandpass filtering between $1000-5 \mathrm{~s}$.

Cluster 2 (Salsa)

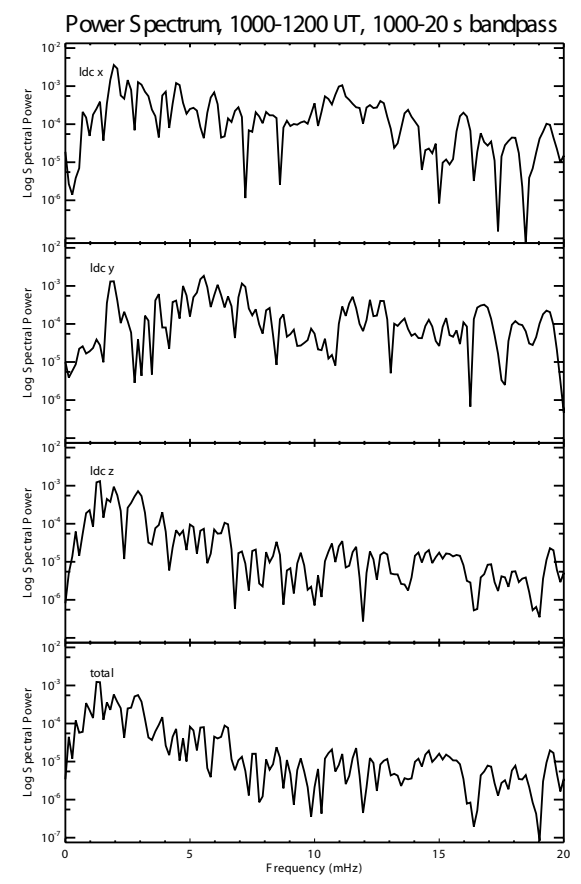

Fig. 7. A Fourier spectrum of the LDC $X, Y, Z$ component and total magnetic field from Cluster 2, from 10:00-12:00 UT, bandpass filtered between 1000-20 s. 
IMAGE 020822

(a) X component

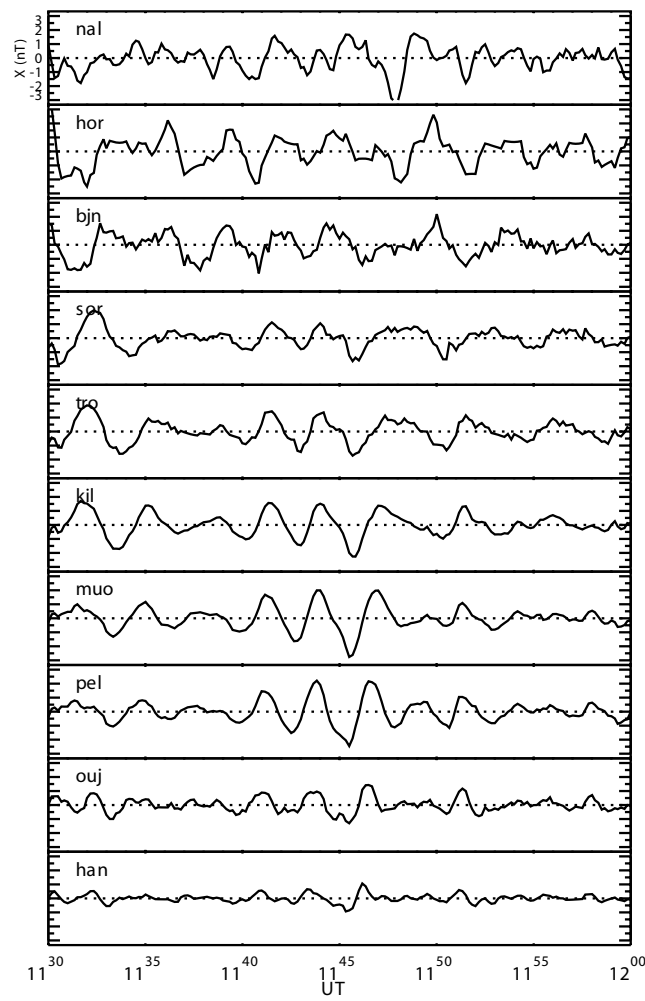

(b) Y component

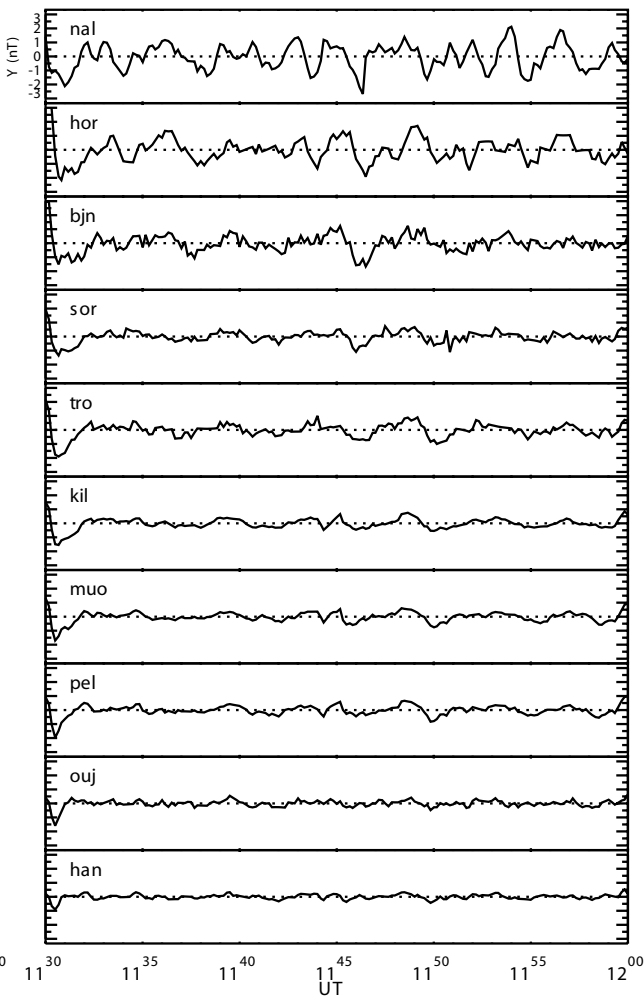

Fig. 8. (a) the $X$ component and (b) the $Y$-component, IMAGE magnetometer data on 22 August 2002 for 11:30-12:00 UT, bandpass filtered between 300-20s.

\section{Discussion}

Cluster magnetic field observations of MHD wave signatures have been investigated from 22 August 2002 when the spacecraft was crossing the dipolar region of the inner magnetosphere, when the footprint location falls in the region of IMAGE-network of ground magnetometers. A spectrogram obtained from Cluster magnetic field variations, presented in Fig. 4 shows an increase in wave frequency in the inbound sector and decrease in frequency in the outbound sector in the transverse components of the local dipole coordinate system during local morning and day time hours. This is the typical characteristic of standing Alfvén waves excited on freely-ringing geomagnetic field lines.

These results are consistent with the AMPTE/CCE observations (Engebretson et al., 1986) in the sense that they obtained the similar results for the toroidal component. The variation of frequency with $L$ in other components as observed by AMPTE/CCE was not that obvious. The signatures of standing Alfvén waves present in both the transverse components suggest that toroidal and poloidal modes are coupled and magnetic shells are oscillating with an arbitrary polarization transverse to the field line. It should be noted that toroidal and poloidal modes are the limiting cases of azimuthal wave numbers, $m$, being 0 and $\infty$ respec-

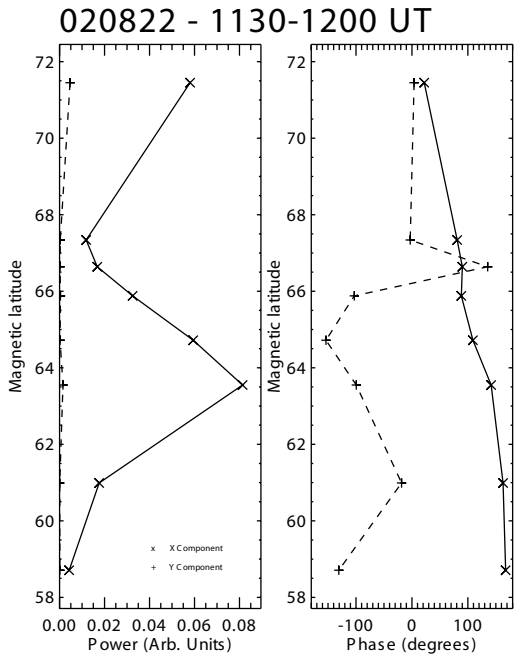

Fig. 9. Latitude profile of the Fourier power and phase of the dominant peak $(6.1 \mathrm{mHz})$ as seen in the power spectra of $X$ and $Y$ components of IMAGE magnetometer data on 22 August 2002 for 11:3012:00 UT, as shown in Fig. 8. The diagram indicates that the power of the dominant peak maximises at around $63.5^{\circ}$ magnetic latitude with a phase reversal at the site of maximum power, the signature of a field line resonance. 
IMAGE 020822

(a) X component

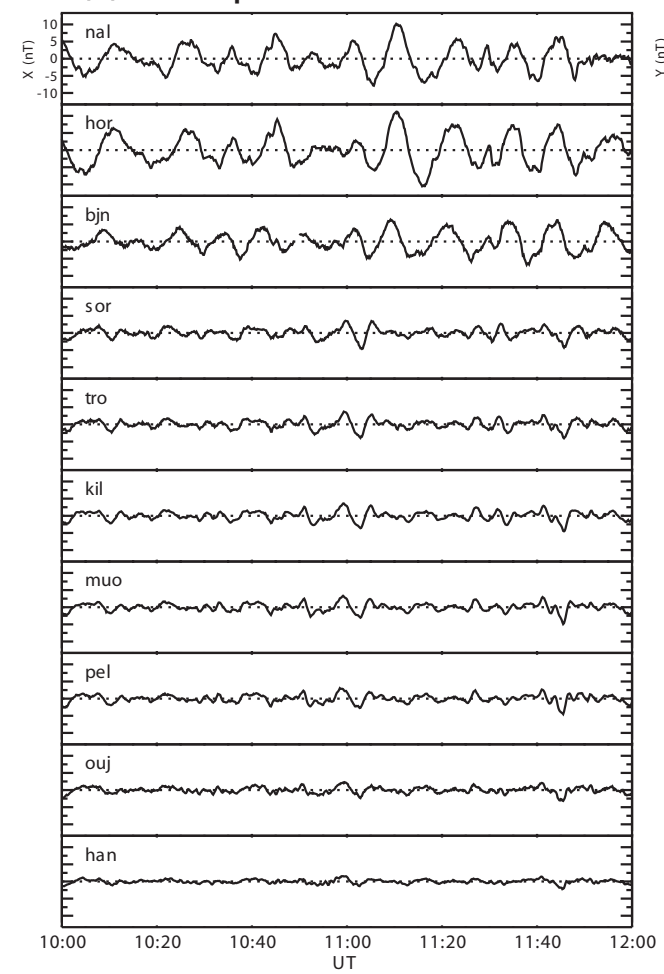

(b) Y component

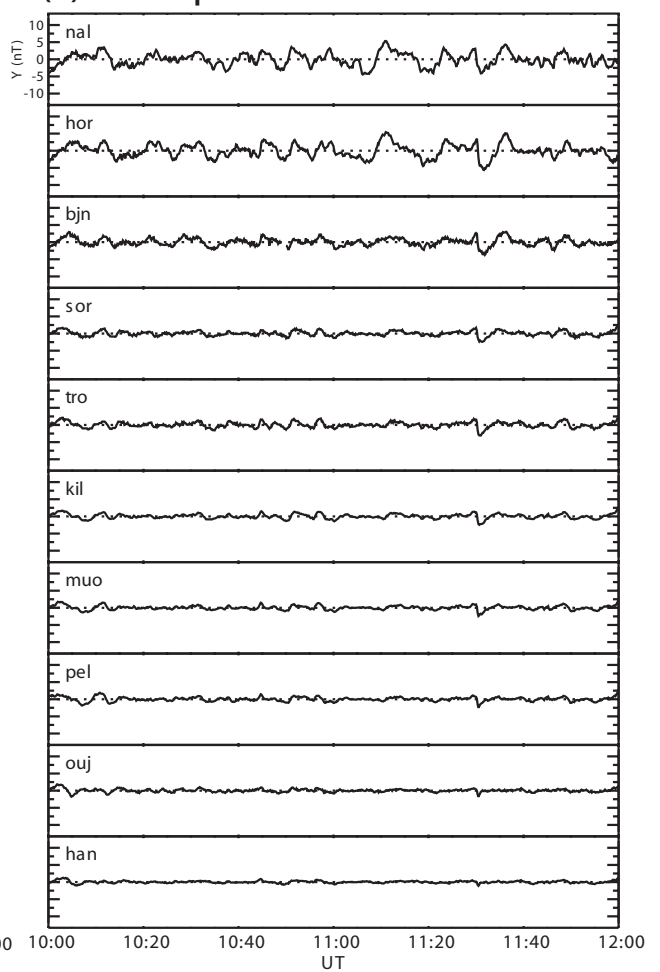

Fig. 10. (a) the $X$ component and (b) the $Y$-component, IMAGE magnetometer data on 22 August 2002 for 10:00-12:00 UT, bandpass filtered between 1000-20 s.

tively, and in a realistic physical situation with finite $m$, they should be coupled (Southwood and Hughes, 1983). If that be the case the signatures of standing Alfvén waves should be seen in both transverse components oscillating at common frequencies. The continuous $L$-variation in the resonant frequency suggests that neighbouring $L$-shells can oscillate with very little interaction in the transverse direction with an arbitrary polarization perpendicular to the field line. Theoretical analysis has suggested that poloidal oscillations should evolve into toroidal oscillations over time (Mann and Wright, 1995; Mann et al., 1997), with the timescale being determined by the azimuthal wavenumber of the oscillation and the Alfvén velocity profile in which it is embedded. Thus it is possible that the oscillations observed here have a higher azimuthal wavenumber than those observed by Engebretson et al. (1986). No evidence of these latitude-dependent signatures are detected in the ground magnetometer data, either in the $10 \mathrm{~s}$ sampled IMAGE data or $1 \mathrm{~s}$ sampled data where it was available (not shown). This is perhaps because these small amplitude, high-frequency signatures are masked by instrument resolution and ionosphereic noise, or could result from higher azimuthal wavenumbers resulting in ionospheric screening (Hughes and Southwood, 1976). Additional events with multispacecraft data from Cluster may be able to address this issue.
In addition to the freely-ringing field line signatures discussed above, a latitude-independent "hot spot" is observed in the dynamic spectrum presented in Fig. 4 just after 11:00 UT. Additional evidence of lower-frequency signatures at 6 and $10-16 \mathrm{mHz}$ are seen in the static spectrum shown in Fig. 5. The conjugate ground data at this time have been presented in Fig. 8, where a $6.1-6.6 \mathrm{mHz}$ peak dominates. The spectral power of this frequency peaks at PEL at a geomagnetic latitude of $63.6^{\circ}$, and shows a latitudinal phase change in the $X$ component magnetic field characteristic of a field line resonance. It thus seems likely that the dominant "hot spot" frequency observed by Cluster is related to this ground signature, and is not the fundamental frequency of the Cluster field line. A model calculation based on Cummings et al. (1969) at $L=5$ with the equatorial hydrogen plasma density $1 \mathrm{~cm}^{-3}$ shows that fundamental frequency for toroidal oscillations would lie between $48 \mathrm{mHz}$ to $60 \mathrm{mHz}$ for a plasma density variation with radial distance which varied as a power law described by a density index lying between 6.0 and 0.0. An equatorial plasma density of the order of $10 \mathrm{~cm}^{-3}$ at this $L$-value is not unusual and would bring the fundamental frequency down to $15-20 \mathrm{mHz}$. Engebretson et al. (1986) used a similar equatorial plasma density to match the observed fundamental frequency with those predicted by the model of Cummings et al. (1969) at $L=5.5$. The fundamental frequency calculated for equatorial plasma density 


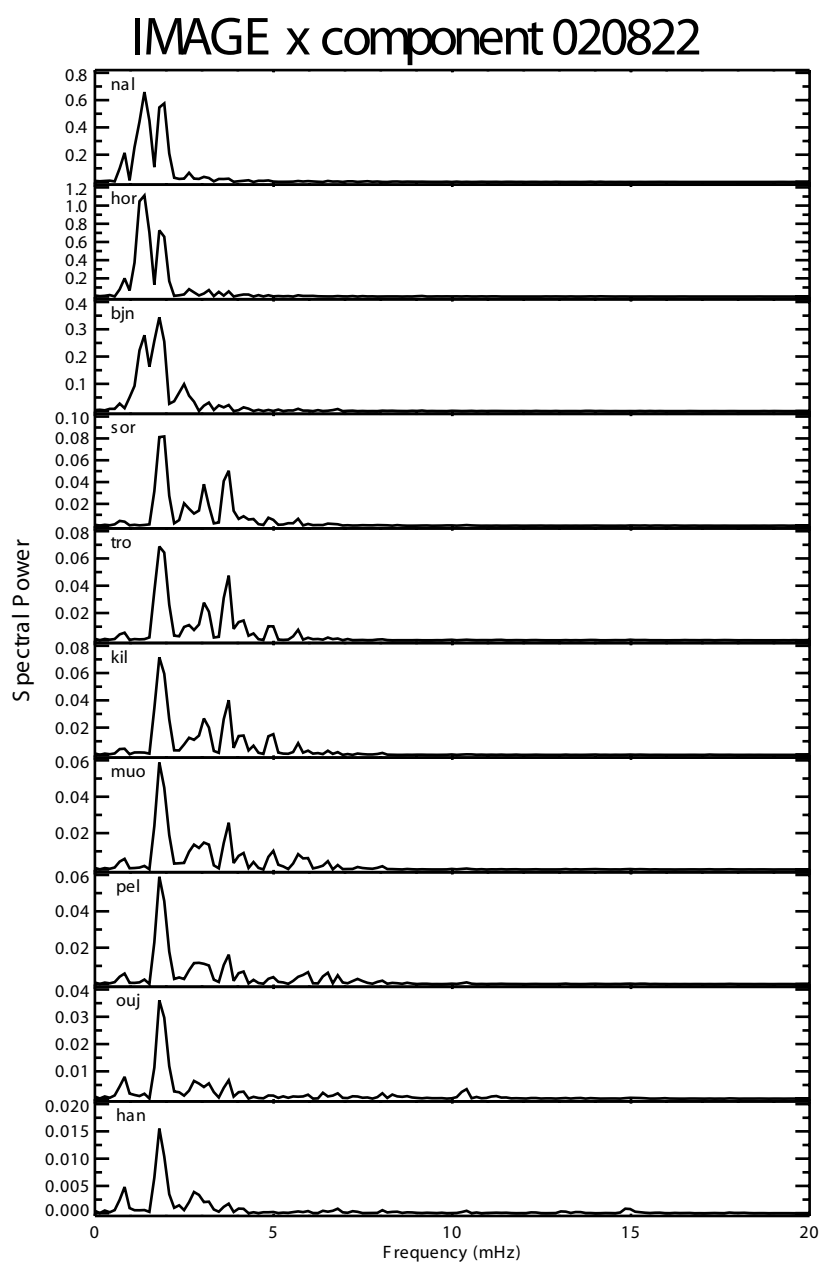

Fig. 11. Fourier spectra of $X$-component IMAGE magnetometer data on 22 August 2002 for 10:00-12:00 UT, bandpass filtered between 1000-20 s.

of 1 per $\mathrm{cm}^{3}$ at $L=5.5$ for density index 3 comes out to be $36.9 \mathrm{mHz}$ and they needed the equatorial plasma density of the order of $10 \mathrm{~cm}^{-3}$ to match to the observed $(11.5 \mathrm{mHz})$ frequency. Fortunately Cluster can provide a direct measurement of the mass density of the plasma. At this time the WHISPER (Décréau et al., 1997) instrument measures an electron density of $20 \mathrm{~cm}^{-3}$, whilst the CIS instrument (Rème et al., 1997) indicates that the plasma population is $\sim 10 \%$ oxygen. These values combine to give a plasma density of $50 \mathrm{amu} \mathrm{cm} \mathrm{cm}^{-3}$. If we use this equatorial plasma density at $L=5$ (11:30 UT), for density index $m=0-6$, the fundamental toroidal frequency as calculated from the Cummings et al. (1969) model comes out to be 5.4-7.6 mHz. Using the same criteria of plasma density the frequencies for second and third harmonic are found to be $14.5-15.7 \mathrm{mHz}$ and $23 \mathrm{mHz}$ respectively. Thus, the observed values of frequencies match quite well with those predicted from the model, provided the dominant ground frequency is taken to be the fundamental, and the dominant "hot spot" frequency observed on Cluster to be the third harmonic. Examining the

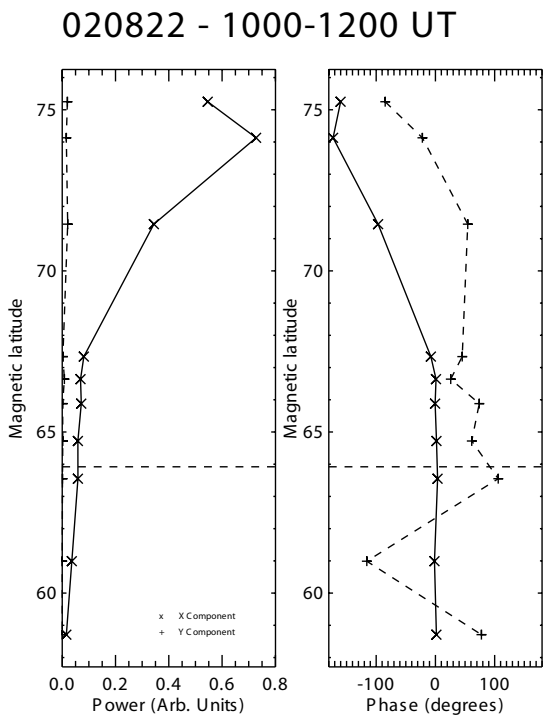

Fig. 12. Latitude profile of the Fourier power and phase of the dominant peak $(1.9 \mathrm{mHz})$ as seen in the power spectra of $X$ and $Y$ components of IMAGE magnetometer data on 22 August 2002 for 10:00-12:00 UT ( $X$ component as shown in Fig. 11). The diagram indicates that the power of the dominant peak maximises at around $74^{\circ}$ magnetic latitude with a phase reversal close to the site of maximum power, the signature of a field line resonance.

analytic solution for the variation of the magnetic field perturbation along the dipolar field lines presented in Sinha and Rajaram (1997) indicates that the Cluster spacecraft are indeed expected to be far from magnetic field antinodes for the first two harmonics, and this may explain the dominance of the third harmonic at this time. Some departure from the predicted value could be due to the fact the Cummings et al. (1969) model was based on the assumption of dipole magnetic field and realistically the field is slightly non-dipolar in nature, as illustrated by the model field presented in Fig. 3.

In addition to the freely-ringing field line signatures and the driven resonant field lines described above, which were Alfvénic in nature, a number of lower frequency compressional peaks have been identified in the Cluster data, with spectral peaks at frequencies of $0.8,1.4,1.9,2.9$, and $3.7 \mathrm{mHz}$. In fact an identical harmonic sequence of low frequency oscillations has been observed in the Cluster compressional components and on the ground magnetometer array (see Figs. 7 and 11, respectively). These observed frequencies are in line with the earlier observations (Kepko and Spence, 2003; Fenrich et al., 1995; Samson et al., 1991; Mitchell et al., 1990) of the so-called magic frequencies of the magnetospheric waveguide modes. An examination of the latitude profile of the Fourier power and phase of one of these lower frequency peaks, at $1.9 \mathrm{mHz}$, has provided evidence of an additional driven field line resonance at higher latitudes than those covered by Cluster (Fig. 12).

The cavity formed by magnetospheric boundaries can be set into oscillation by impulsive driving such as solar wind pressure variation (Menk et al., 2003) or broad 
band driver such as Kelvin-Helmholtz waves (Chen and Hasegawa, 1974b; Mann et al., 1999). Such oscillations of the magnetospheric waveguide might then cause a local field line to oscillate at its natural frequency where this frequency matches one of the waveguide harmonics (Harrold and Samson, 1992; Samson et al., 1992; Walker et al., 1992; Wright, 1994; Rickard and Wright, 1994). It was revealed through our ground and spacecraft observation that the waveguide oscillations showing discrete as well as harmonically related frequencies were in operation during the period when the field line oscillation event was identified through satellite and ground observations. Though the lower frequency waveguide modes dominate the power spectra, there is a hint of higher frequency oscillations especially at the stations of relatively lower latitudes. These oscillations could either be discrete or harmonically related to those dominating the lower frequency band. These higher frequency waveguide modes are of relatively smaller amplitudes compared to those seen at lower frequencies and don't show significant power. However, if their frequency matches with the natural frequency of local field lines, they can cause the field lines to oscillate at their characteristic frequency. Because of the fact that the waveguide oscillations are in operation during the event, we believe that higher frequency modes (whose power are not significant in the observed spectra) might be playing a role in exciting field line resonance (frequency $\sim 6 \mathrm{mHz}$ ) at those latitudes.

In conclusion, we can say that the $L$-variation of pulsation frequency observed in the satellite supplemented by the fundamental frequency observed on the ground essentially supports the theory of field line oscillations as a viable mechanism for the observed geomagnetic pulsations as initially proposed by Dungey (1954) and latter supported by many theoretical and observational studies (Cummings et al., 1969; Anderson et al., 1990 and references therein). There is also evidence of these modes being excited due to the coupling of cavity/waveguide oscillations as suggested by Zhu and Kivelson (1988). This is the first investigation of its type from Cluster combined with ground observation that supports the theory of field line oscillations. It should be noted that the satellite like Cluster provides a unique opportunity of detecting these field line oscillations because it consists of four spacecraft which might track different geomagnetic field line as is evident from Fig. 3. All of them might not lie in the favourable dipolar region and hence miss the event, but there is a greater possibility of observing these events by one spacecraft or the other, and determining the harmonic structure along the field lines. We have done extensive analysis of the cluster magnetic field data when the spacecraft was crossing the dipolar inner magnetosphere and the detailed investigations to put the excitation mechanism of these resonant oscillations in proper perspective is the subject of our ongoing studies and will be communicated in due course.

Acknowledgements. The authors are grateful for the provision of contextual data from the WHISPER and CIS instruments on board the Cluster satellites. We thank P. M. E Décréau and H. Rème, the respective PIs of these instruments, for allowing us access to the data and their helpful contributions.

Topical Editor T. Pulkkinen thanks A.Wright and another referee for their help in evaluating this paper.

\section{References}

Allan, W. and Poulter, E. M.: The spatial structure of different ULF pulsation types: A review of STARE radar results, Rev. Geophys., 22, 85-97, 1984.

Allan, W., White, S. P., and Poulter, E. M.: Magnetospheric coupling of hydromagnetic waves- initial results, Geophys. Res. Let., 12, 287-290, 1985.

Allan, W., White, S. P., and Poulter, E. M.: Impulse-excited hydromagnetic cavity resonances in the magnetosphere, Planet. Space Sci., 34, 371-385, 1986a.

Allan, W., White, S. P., and Poulter, E. M.: Magnetospheric wave coupling in the magnetosphere- plasmapause effects on impulseexcited resonances, Planet. Space Sci., 34, 1189-1200, 1986 b.

Allan, W., White, S. P., and Poulter, E. M.: Hydromagnetic wave coupling in the magnetosphere - magnetic fields and Poynting fluxes, Planet. Space Sci., 35, 1181-1192, 1987a.

Allan, W., White, S. P., and Poulter, E. M.: Structure of magnetospheric MHD resonances for moderate "azimuthal" asymmetry, Planet. Space Sci., 35, 1193-1198, 1987b.

Anderson, B. J., Engebretson, M. J., and Zanetti, L. J.: Distortion effects in spacecraft observations of MHD toroidal standing waves: theory and observations, J. Geophys. Res., 94, 13425$13445,1989$.

Anderson, B. J., Engebretson, M. J., Rounds, S. P., Zanetti, L. J., and Potemra, T. A.: A statistical study of Pc3-5 pulsations observed by the AMPTE/CCE magnetic fields experiment 1. Occurrence distributions, J. Geophys. Res., 95, 10 495-10 523, 1990.

Chen, L. and Hasegawa, A.: A theory of long-period magnetic pulsations- 1. Steady state excitation of field line resonances, J. Geophys. Res., 79, 1024-1032, 1974a.

Chen, L. and Hasegawa, A.: A theory of long-period magnetic pulsations- 2. Impulse excitation of surface eigenmode, J. Geophys. Res., 79, 1033-1037, 1974b.

Crowley, G., Hughes, W. J., and Jones, T. B.: Observational evidence of cavity modes in the Earth's magnetosphere, J. Geophys. Res., 92, 12 233-12 240, 1987.

Crowley, G., Hughes, W. J., and Jones, T. B.: Correction to "Observational evidence of cavity modes in the Earth's magnetosphere", J. Geophys. Res., 94, 1555-1555, 1989.

Cummings, W. D., O'Sullivan, R. J., and Coleman, P. J.: Standing Alfvén waves in the magnetosphere, J. Geophys. Res., 74, 778793, 1969.

Décréau, P. M. E., Fergeau, P., Krasnoselskikh, V., Lévêque, M., Martin, P., Randriamboarison, O., Sené, F. X., Trotignon, J. G., Canu, P., Mögensen, P. B., and Whisper investigators: Whisper, a resonance sounder and wave analyser: performances and perspectives for the Cluster mission, Space Sci. Rev., 79, 157-193, 1997.

Dungey, J. W.: Electrodynamics of the outer atmospheres, Rep. 69, Ions. Res. Lab. Pa. State Univ., University Park, 1954.

Engebretson, M. J., Zanetti, L. J., Potemra, T. A., and Acuña, M. H.: Harmonically structured ULF pulsations observed by AMPTE/CCE magnetic field experiment, Geophys. Res. Let., 13, 905-908, 1986. 
Fenrich, F. R., Samson, J. C., Sofko, G., and Greenwald, R. A.: ULF high- and low-m field line resonances observed with the Super Dual Auroral radar Network, J. Geophys. Res., 100, 21 53521547,1995

Hapgood, M. A.: Space physics coordinate transformations: A user guide, Planet. Space Sci., 40, 711-717, 1992.

Harrold, B. G. and Samson J. C.: Standing ULF modes of the magnetosphere,: a theory, Geophys. Res. Lett., 19, 1811-1814, 1992.

Hughes, W. J. and Southwood, D. J.: The screening of micropulsation signals by the atmosphere and ionosphere, J. Geophys. Res., 81, 3234-3240, 1976.

Kepko, L. and Spence, H. E.: Observations of discrete, global, magnetospheric oscillations directly driven by solar wind density variations, J. Geophys. Res., 108, 1257, doi:10.1029/2002JA009676, 2003.

Kivelson, M. G. and Southwood, D. J.: Resonant ULF waves: A new interpretation, Geophys. Res. Let., 12, 49-52, 1985.

Kivelson, M. G. and Southwood, D. J.: Coupling of global magnetospheric MHD eigenmodes to field line resonances, J. Geophys. Res., 91, 4345-4351, 1986.

Kivelson, M. G., Etcheto, J., and Trotignon, J. G.: Global compressional oscillations of the terrestrial magnetosphere: The evidence and a model, J. Geophys. Res., 89, 9851-9856, 1984

Mann, I. R. and Wright, A. N.: Finite lifetimes of ideal poloidal Alfvén waves, J. Geophys. Res., 100, 23 677-23 686, 1995.

Mann, I. R., Wright, A. N., and Hood, A. W.: Multiple-timescales analysis of ideal poloidal Alfvén waves, J. Geophys. Res., 102, 2381-2390, 1997.

Mann, I. R., Wright, A. N., Mills, K. J., and Nakariakov, V. M.: Excitation of magnetospheric waveguide modes by magnetosheath flows, J. Geophys. Res., 104, 333-353, 1999.

McDiarmid, D. R. and Nielsen, E.: Simultaneous observation of monochromatic and variable period geomagnetic pulsations, J. Geophys. Res., 92, 4449-4457, 1987.

Menk, F. W., Yeoman, T. K., Wright, D. M., Lester, M., and Honary, F.: High-latitude observations of impulse-driven ULF pulsations in the ionosphere and on the ground, Ann. Geophys., 21, 559576,2003

\section{SRef-ID: 1432-0576/ag/2003-21-559.}

Menk, F. W., Waters, C. L., Ziesolleck, C. W. S., Fraser, B. J., Lee, S. H., and McNabb, P. W.: Ground measurements of low latitude magnetospheric field line resonances, in: Solar wind sources of magnetospheric ULF waves Eds. Engebretson, M. J., Takahashi K., and Scholer, M., Geophys. Mono., 81, AGU, Washington D.C., 299-308, 1994.

Mitchell, D. G., Engebretson, M. J., Williams, D. J., Cattell, C. A., and Lundin, R.: Pc5 pulsations in the outer dawn magnetosphere seen by ISEE 1 and 2, J. Geophys. Res., 95, 967-975, 1990.

Poulter, E. M., Allan, W., and Bailey, G. J.: ULF pulsation eigenperiods within the plasmosphere, Planet. Space Sci., 36, 185-196, 1988

Poulter, E. M. and Nielsen, E.: The hydromagnetic oscillations of individual shells of geomagnetic field, J. Geophys. Res., 87, 10 432-10 438, 1982.

Poulter, E. M. and Allan, W.: Transient ULF pulsation decay rates observed by ground based magnetometers: The contribution of spatial integration, Planet. Space Sci., 33, 607-616, 1985.
Rème, H., Bosqued, J.-M., Sauvaud, J.-A. et al.: The Cluster Ion Spectrometry (CIS) Experiment, Space Sci. Rev., 79, 303-350, 1997.

Rickard, G. J. and Wright, A. N.: Alfvén resonance excitation and fast wave propagation in magnetospheric waveguides, J. Geophys. Res., 99, 13 455-13 464, 1994.

Rostoker, G. and Samson, J. C.: Pc micropulsations with discrete, latitude-dependent frequencies, J. Geophys. Res., 77, 62496243, 1972.

Samson, J. C. and Rostoker, G.: Latitude-dependent characteristics of high latitude Pc4 and 5 micropulsations, J. Geophys. Res., 77, 6133-6144, 1972.

Samson, J. C., Jacobs, J. A., and Rostoker, G.: Latitude-dependent characteristics of long-period geomagnetic micropulsations, J. Geophys. Res., 76, 3675-3683, 1971.

Samson, J. C., Greenwald, R. A., Ruohoniemi, J. M., Hughes, T. J., and Wallis, D. D.: Magnetometer and radar observations of magnetohydrodynamic cavity modes in the earth's magnetosphere, Can. Jour. Phys., 69, 929-937, 1991.

Samson, J. C., Harrold, B. G., Ruohoniemi, J. M., and Walker, A D. M.: Field line resonance associated with MHD waveguides in the magnetosphere, Geophys. Res. Lett., 19, 441-444, 1992.

Sinha, A. K., Pathan, B. M., Rajaram, R., and Rao, D. R. K.: Low frequency modulation of transionospheric radio wave amplitude at low-latitudes: Possible role of field line oscillations, Ann. Geophys., 20, 69-80, 2002, SRef-ID: 1432-0576/ag/2002-20-69.

Sinha, A. K. and Rajaram, R.: An analytic approach to toroidal eigen mode, J. Geophys. Res., 102, 17 649-17 657, 1997.

Southwood, D. J.: Some features of field line resonances in the magnetosphere, Planet. Space Sci., 22, 483-491, 1974.

Southwood, D. J. and Hughes, W. J.: Theory of hydromagnetic waves in the magnetosphere, Space Sci. Rev., 35, 301-366, 1983.

Takahashi, K. and McPherron, R. L.: Standing hydromagnetic waves in the magnetosphere, Planet. Space Sci., 32, 1343-1359, 1984.

Takahashi, K. and Anderson, B. J.: Distribution of ULF-energy ( $f<80 \mathrm{mHz}$ ) in inner magnetosphere: a statistical analysis of AMPTE CCE magnetic field data, J. Geophys. Res., 97, 10 751$10773,1992$.

Walker, A. D. M.: Coupling between waveguide modes and field line resonances, J. Atmos. Sol.-Terr. Phys., 62, 799-813, 2000.

Walker, A. D. M., Greenwald, R. A., Stuart, W. F., and Green, C. A.: STARE auroral radar observations of Pc5 geomagnetic pulsations, J. Geophys. Res., 84, 3373-3388, 1979.

Walker, A. D. M., Ruohoniemi, J. M., Baker, K. B., and Greenwald, R. A.: Spatial and temporal behaviour of ULF pulsations observed by the Goose Bay HF radar, J. Geophys. Res., 97, 12 187$12202,1992$.

Wright, A. N.: Dispersion and wave coupling in inhomogeneous MHD waveguides, J. Geophys. Res., 99, 159-167, 1994.

Zhu, X. M. and Kivelson, M. G.: Analytic formulation and quantitative solutions of the coupled ULF wave problem, J. Geophys. Res., 93, 8602-8612, 1988. 